Case Reports

\title{
Lessons Learned: Barriers to Hemoglobin A1c Monitoring in Rural Northern Tanzania
}

\author{
Andrea Fortson Knopp and Erika Metzler Sawin \\ School of Nursing, James Madison University, USA
}

\author{
Article history \\ Received: 07-09-2020 \\ Revised: 04-12-2020 \\ Accepted: 12-12-2020 \\ Corresponding Author: \\ Andrea Fortson Knopp \\ School of Nursing, James \\ Madison University, USA \\ Email: knoppaf@jmu.edu
}

\begin{abstract}
In sub-Saharan countries, Non-communicable Chronic Diseases (NCDs), including diabetes, are on the rise. Glycated hemoglobin (HbA1c) is a Point Of Care (POC) diagnostic tool for diabetic monitoring that is unavailable in many resource-constrained environments worldwide. Theoretically, HbA1c measurement is practical in these settings because fasting is not required, the test is not generally repeated before 120 days and travel time for multiple appointments is reduced. To improve diabetes management of patients and the provider's ability to manage chronic diabetes, two HbA1c monitoring machines were used to determine the effectiveness and feasibility of HbAlc in diabetes management in two hospital locations in Tanzania. A follow-up team returned to the sites to determine the use of the $\mathrm{HbA} 1 \mathrm{c}$ machines in the treatment of diabetes and introduced the use of Point Of Care (POC) HbA1c machines. Interviews were conducted with diabetic participants $(n=4)$ regarding their diabetes management experiences. Though short-term change had mixed success, the long-term impact on the management of diabetes is unknown. Cultural influences and access issues had a significant impact on the usefulness of the $\mathrm{HbA} 1 \mathrm{c}$ machines for monitoring diabetes in rural northern Tanzania. A greater understanding of these factors is needed in order to creatively address these barriers. The Tanzania HbA1c project functions as a case study at a population level through which to examine the complexities of diabetes management in a resource-constrained environment.
\end{abstract}

Keywords: Diabetes, International Health, Primary Care, Rural Health

\section{Introduction}

Tanzania is a country in the midst of epidemiological transition. Tuberculosis, malaria, lower respiratory infections and HIV are leading causes of death; however, Non-Communicable Disease (NCDs) such as ischemic heart disease, diabetes and stroke are becoming more prevalent (CDC, 2019). NCDs were once considered rare in low income countries like Tanzania but since the 1980s they have been on the rise (Kolling et al., 2010). NCDs are disproportionately represented in Low-and Middle-Income Countries (LMICs), with $80 \%$ of the 415 million individuals living with diabetes worldwide residing in LMICs (Ndayisaba et al., 2017). In Tanzania, Type II diabetes prevalence is rising and is uniquely complicated by the presence of endemic infectious diseases, creating a prime example of the double disease burden plaguing LMICs globally (Nnko et al., 2015). NCD-related data collection in all of sub-Saharan Africa is poor, but prevalence estimates of diabetes in Tanzania range from $4.3 \%$ (WHO, 2016) to $9.1 \%$ (WHO, 2012). Regardless of the variation, diabetes in Tanzania is a growing problem, one that a low-resource country with a double disease burden is poorly equipped to handle.

Hemoglobin A1c (HbA1c) Point Of Care (POC) testing machines are well-known, well-respected and routinely used for both diagnosing and monitoring diabetes. A collaboration between a USA-based university and two hospitals to introduce these machines to two areas in Tanzania was established and involved creating Tanzania-based teams with a focus on education and sustainability. Siemens Hemoglobin analyzers were chosen with an eye on sustainability, since supplies, parts and kits were available in-country. A liaison was established at each hospital to ensure continuity of this project. The purpose of this study is to (1) describe the Tanzania HbA1c project; (2) explore the use of HbA1c machines in low-resource countries and (3) suggest evidence-based solutions and ideas for future collaboration. 


\section{Review of the Literature}

\section{Chronic Disease Management in Low-resource Countries, Including Tanzania}

NCD burden disproportionately affects LMICs such as Tanzania and adversely shapes health outcomes (Stanifer et al., 2016). For instance, sub-Saharan Africa has the highest diabetes-related mortality rate in the world (Park and Pastakia, 2018). Typically, although there are many health initiatives focusing on infectious/ communicable diseases; there are few programs dedicated to NCDs, particularly in rural areas (Mwangome et al., 2016). Care is generally provided at district or regional facilities, which are far-reaching and difficult to access and primary care is often fragmented and not continuous (Mwangome et al., 2016). Nnko et al. (2015) noted that NCD medications are costly and often out of stock and that patient often are charged for treatment, although charges are rarely collected.

Meiqari et al. (2019) found that the Western concepts of chronic disease management and continuity of care are largely unknown and non-existent because they rely on a universally accessible and well-organized primary care base, often absent in resource-constrained settings (Meiqari et al., 2019). Mwangome et al. (2016) noted that NCD service organization and delivery modalities affect access, acceptance and utilization. In parts of Tanzania, NCD care is disorganized and patient understanding of NCDs is reportedly gleaned from low-information health facility visits and uninformed anecdotal information, which may be why patients often do not adopt disease-reducing behaviors or seek NCD care (Nnko et al., 2015). Because NCDs involve a modifiable behavior component, some providers blame patients for having an NCD, leading to decreased patient trust (Nnko et al., 2015). Additionally, rural Tanzania has a health care worker shortage, affecting care access and quality (Mwangome et al., 2016).

\section{Diabetes in Low-Resource Countries Including Tanzania}

Diabetes incidence rates are increasing, in part due to increased life expectancy (Peer et al., 2014). The (MHSW, 2008) developed an action plan for NCD prevention and control. The diabetes-specific plan includes operationalized evidence-based guidelines, a referral protocol and a diabetes registry plan. It specifies that the minimum staffing requirement at primary health care diabetes service sites includes a nurse, a village health worker, a doctor and a diabetes educator (Peer et al., 2014). Additionally, private international diabetes interest groups are contributing to community awareness and advocacy through strategic plan creation (WDF, 2016).
Mwangome et al. (2017) found that diabetes care, like other NCD care, was centralized versus localized, which participants noted is not conducive to high-quality chronic disease care. Expecting rural, low-income patients to travel to distant referral hospitals for the rest of their lives is unrealistic (Park and Pastakia, 2018). Participants stated that diabetes training, management and reporting systems were weak, especially in rural Tanzania and that there were inadequate guidelines and medications (Mwangome et al., 2017). Providers felt illprepared to perform adequate diabetes care, due to low diabetes management knowledge as well as unreliable sources of medical equipment. In the (Mwangome et al., 2017), only one large referral hospital had diabetes education materials and those were developed by nonresidents. Only one provider recounted reading and giving them to patients. Because many rural Tanzanian facilities lack the equipment and services needed for diabetes prevention and management, many individuals either at-risk for or with diabetes are frequently undiagnosed or untreated (Stanifer et al., 2016; Nnko et al., 2015). Lastly, although metformin may be available, insulin, which requires refrigeration, is not an option for many rural residents (Peer et al., 2014).

Basic knowledge about diabetes and NCDs in general is lacking. Participants in a Tanzania-based study described chronic diseases as 'any disease which is incurable' and 'only temporarily relieved by drugs"” (Nnko et al., 2015). Causes of diabetes were attributed to excessive consumption of fatty, sugary and salty foods, although some participants mentioned other causes of diabetes such as witchcraft or environmental pollution (Nnko et al., 2015). Most participants reported that they thought patients with diabetes frequently pass sugary urine, based on the observation that diabetics' urine attracted ants. Indeed, the Swahili word for diabetes is 'kisukari,' from the Swahili word for sugar, 'sukari' (Nnko et al., 2015).

\section{HbAlC Point-of-Care Testing}

$\mathrm{HbA} 1 \mathrm{c}$ POC testing is indisputably the best method for testing blood glucose level and can function well in resource-constrained environments because it is a onetime test providing immediate feedback about average glucose levels over time, thereby improving control (Park and Pastakia, 2018; Balde et al., 2017; Stanifer et al., 2016). Camara et al. (2015) found a correlation between lack of $\mathrm{HbA1c}$ testing and poor glycemic control in Guinea. However, there are issues with $\mathrm{HbA} 1 \mathrm{c}$ machines. Both (Camara et al., 2015; Balde et al., 2017) noted problems with affordability and access. Machine maintenance, staff training, laboratory setting availability and difficult-to-access reagents (some of which have to be refrigerated) can be difficult in LMICs (Drain et al., 2014). Strict quality assurance and alignment with international reference values is necessary to ensure accuracy (Drain et al., 2014). Cost benefit is imperative (Drain et al., 2014). 
The long-term benefit of hyperglycemia screening is questionable, even in high income countries and there is no evidence supporting screening in LMICs (Atun et al., 2017). Due to high cost and limited availability, is it unlikely that $\mathrm{HbA} 1 \mathrm{c}$ testing can be consistently utilized in LMIC primary care facilities (Atun et al., 2017; Bergman et al., 2012). However, other diabetes testing methods such as urine strips for glucose and ketone measurement, oral glucose tolerance tests and blood glucose measurement exist (WHO, 2016). Lastly, HbA1c testing faces challenges in sub-Saharan Africa because a whole red blood cell sample is needed, which can be affected by malaria and sickle cell disease (Atun et al., 2017; Bergman et al., 2012).

\section{Materials and Methods}

\section{Design}

A USA-based School of Nursing has been involved in northern Tanzania as part of the university's international programs for several years. In the interests of deepening impact for both American students and Tanzanian communities, a faculty research team performed a needs assessment in the Summer of 2016 in the rural underserved areas of Shirati (Mara Region) and Moshi (Kilimanjaro Region). The researchers visited hospitals and spoke with providers and administrators to determine the greatest needs. Medical hospital directors in both regions expressed a desire for $\mathrm{HbA} 1 \mathrm{c}$ machines to improve diabetes diagnosis and management. Neither hospital laboratory had a HbA1c machine, which were rare in Tanzania.

In 2017, after receiving university IRB approval, two HbA1c machines were introduced, one at each hospital. The analyzers were purchased with internal university grant funding. To ensure sustainability and usage, researchers determined that Siemens HbA1c analyzers were the most appropriate option, since additional supplies, parts and kits can be obtained in Tanzania, eliminating cost-prohibitive shipping expenses. Implementation teams included graduate and undergraduate healthcare students participating in a summer international education program, who were provided with education on cultural influences in Tanzania and the local Swahili language. In-country implementation began with general presentations on the use of the HbA1c machines, including a demonstration with a test patient and several focused training sessions with those who would be primary machine users. The target audience was key hospital stakeholders, including the chief medical officer, medical doctors, clinical officers, nurses and lab technicians. The presentation consisted of evidence-based information about $\mathrm{HbA} 1 \mathrm{c}$ monitoring and details on machine operation. Additional training sessions were tailored to each discipline's practice scope. After training completion, hospital staff trialed the HbA1c machines, with the American student team available for trouble shooting.

In 2018, the researchers returned to the sites to determine the effectiveness and use of the HbA1c machines. Researchers interviewed diabetic participants who had their HbA1c checked with the machines at one of the two locations. Interviews consisted of five questions, used to initiate participants' experiential narratives (Table 1) and were conducted on location at the hospital or clinic in a private location, or in participants' homes. The participants provided either verbal or written consent prior to the interviews. The interviews were audio recorded using Swahili interpreters. The interviews were transcribed by the main author and analyzed using NVIVO.

In 2019, nursing faculty researchers and undergraduate nursing students performed a follow-up study, in which several glycosylated Hemoglobin (HbA1c) Point Of Care (POC) diagnostic tools were brought to additional rural locations, to both a hospital in the Mara District and to a community based clinic in the Kilimanjaro region to introduce the use of $\mathrm{Hb} \mathrm{Alc}$ machines in the management of diabetes. However, when the group returned, the relationship with the Kilimanjaro Hospital was non-existent and it was clear that the HbA1c device was not being used routinely, if at all, in the Mara Region, served mainly by Shirati Hospital. Although the hospital itself contains 150 beds and operates four clinics, they do not have a dedicated diabetes care or prevention program (Wetzel, 2011). Even though enthusiasm and learning ability were present upon initial project implementation, institutional readiness and capacity were not and are needed for successful incorporation of the HbA1c machines into clinical practice long term.

At this time, the authors will describe the results of the 2018 qualitative interviews and then analyze the HbA1c project overall, with a view to envision sustainable future collaboration.

Table 1: Interview questions and probes

Tell me about how you learned you had diabetes? What was that experience like?

What is it like for you to have diabetes?

How do you take care of your diabetes?

Have you done anything different to take care of your diabetes in the last year?

What has the doctor told you about your diabetes and how to take of yourself? 


\section{Results}

Researchers conducted interviews with Tanzanians with diabetes who had had experience with the HbA1c machines $(n=4)$ selected randomly from the records of $\mathrm{HbAc}$ at the clinic. Using descriptive analysis, interview data was coded into themes. Based on the interviews, three main themes were identified: Anxiety/fear, adaptation of information and cultural impediments to a diabetes-focused approach to diet and exercise.

When describing what it is like having diabetes in rural Tanzania, participants reported it is "disturbing" and "I'm afraid to do what I was not told to do," indicating that a diagnosis of diabetes incited fear and anxiety. Exacerbating the fear and anxiety was misunderstanding and misinformation about the disease process. One woman stated "People with diabetes usual to have fat, but me I'm thin." Another patient stated she was told "up to now don't do any sex activity" and "stop working". Participants also noted surprise at the suddenness of having diabetes. Two women commented "Suddenly, .. I am having this disease...suddenly I got it" and "it [diabetes] was appearing suddenly at that time."

Adaptation of information to having diabetes was seen in patients attending the diabetes clinic for their medicine and diabetes education. The clinic was both a source of misinformation but also some sound information about healthier eating and exercise. Participants reported learning about symptoms of elevated blood glucose. They also described what they should eat such as "what she does now is use much not sugar, take more little bites in the food." Almost all the participants reported using medication and checking glucose levels when possible, at the hospital lab.

Cultural issues appeared to be in conflict with some of the diabetes education, particularly related to eating and activity. Alcohol use was common among the participants and the advice to stop being an "alcohol taker" was difficult to adopt. One participant was told "to do some exercises to keep her body in a good way" but now she has back pain when she walks and ... "can't dig." This was problematic for this woman in particular, since she farms and gathers food. Also, the advice given to participants to eat "white food" such as chicken, fish and meat was also alluded to as white person's food. One woman explained:

"The problem is that I don't have options here...in the shops. We don't have the special rice for diabetic cases...here. There's a special bread, a brown bread which is for diabetic cases, (that) we don't have here...But at least I am trying to avoid ...the foods with a lot of carbohydrates. But I eat rice, just normal rice. I have no choice".
The Western ideas of "special" diabetic food, not based on available Tanzanian food and exercise requirements above and beyond work were barriers to diabetic self-management. The idea of "exercising" separate from rural Tanzanians' already labor-intensive work creates a perception of having to "do more" to be able to manage diabetes. A participant used the word "fail" in relation to his diet, reflecting a sense of inadequacy about diabetes self-management.

One woman with diabetes explained it this way, regarding a woman that she knows:

“..her tribe, they are Chaggas, so they used to cut the grass for cows or with cattle (s), so she checked with some exercise by walking, by walking on the farm, again she used to cut grass for the cows, so...she gets the rigorous exercise. Do you see? So she makes the exercise."

Asking this participant to "exercise" more would be an unreasonable burden; she already labors all day to provide for her family. This participant was also told to stop eating fish "tacos" and having sexual activity. All of this medical advice is in direct opposition to her societal role and represents a conflict among gender roles, daily responsibilities and diabetes management. Attitudes and beliefs identified through the interviews also affected HbA1c machine use. Fear and anxiety created a barrier to the participants' and other diabetic patients' willingness to be tested. Medical misinformation, such as being told not to have sex, led to participant unwillingness to engage in diabetes management and a lack of follow-up with HbA1c testing.

\section{Discussion}

The themes suggest that the problem of how to prevent, diagnose and manage diabetes is deep, going well beyond HbA1c testing. Regardless of the accuracy of the participants' understanding of diabetes, the result was miscommunication, anxiety and cultural conflict. Successful patient engagement in diabetes management, including the HbA1c machine, is difficult in light of limited access and resources. There is clearly a need for carefully edited, culturally appropriate educational materials. Barriers to diabetes management are complex, stemming from the conceptualization of primary care and chronic diseases in rural Tanzania.

Throughout this project many insights became clear related to the sustainability and effectiveness of the project. A three-week long presence once a year makes it almost impossible to provide the hands-on mentoring that is required for such an endeavor. Although the American project implementers wished to collect data on tests performed and follow-up $\mathrm{HbA1c}$ measurements, 
testing rates were very low, virtually non-existent in 2018. The primary barrier to HbA1c analyzers was obtaining the needed testing supplies due to limited resources. Communication between the hospital and the research team three weeks after the 2019 visit confirmed that the Tanzanian hospital staff remained optimistic that supplies would be obtained and that the machines would eventually be fully incorporated into practice. They reiterated that change in Tanzania takes time and expressed thanks for the new equipment. However, the lack of available follow-up information and data limited analysis nonetheless rendered knowledge of the longterm impact of the HbAlc machines on diabetes management to be unknown.

Although the HbA1c implementation program could be viewed as a failure, there is nonetheless much that can be learned. Access to data on provider usage and testing frequency would help determine which barriers remain. Ongoing conversations with the Tanzanian healthcare workers related to expectations and needs would also contribute to program success. A recommendation would be for a team member or designee to revisit the hospitals, acquiring follow-up statistics related to the acquisition of supplies, number of tests performed and resultant change in treatment. In addition to follow-up visits, it became evident that educational sessions for all providers and clinical officer students were needed in the 2-4 months after the completion of team training. Sessions could consist of presentations with either group or individual case studies for the providers. Such sessions would affirm valuable treatment options for providers based on HbA1c results and would emphasize community benefits.

Cultural influences must be considered when seeking to understand the issue of diabetes care in rural Tanzania. Kolling et al. (2010) noted the importance of acknowledging that not only is diabetes prevalence rising in Tanzania, this is disproportionately affecting the poor. Their urban study participants believed that diabetes is neglected in the public sphere compared to HIV/AIDs, which garners much attention and has free treatment. Kolling et al. (2010) determined that this is more pronounced in rural Tanzania. Indeed, extreme poverty is a cultural factor in Shirati, where the majority of residents have no health insurance for health care services (Shirati KMT Hospital, 2020). Subsistence farmers in the region make the equivalent of 40 USD per month (Peprah et al., 2019). Approximately $80 \%$ of the population in the region lives below the United Nations Poverty Line (Shirati KMT Hospital, 2020) waiting for NCD care can translate into lost wages or potential employment opportunities (Some et al., 2016).

Diabetic Tanzanian study participants in a study by (Nnko et al., 2015) were extremely poor, underweight subsistence farmers. Patients like this do not present with the typical risk factors of obesity and alcohol and/or tobacco use (Ndayisaba et al., 2017). Overweight and obesity is culturally significant throughout sub-Saharan Africa, signifying wealth and happiness. Over nutrition also represents not having HIV, important in a country like Tanzania with such a high HIV prevalence (Peer et al., 2014). Increased access to sugary sodas and processed foods high in salt and fat is problematic, particularly in a culture that values over nutrition. It is important in Tanzanian diabetes education to emphasize sorghum, found in the Tanzanian staple ugali, as a healthy alternative to rice and maize (Mwangome et al., 2017).

The small number of study participants is a limitation. It likely indicates reluctance to talk about diabetes and its impact on daily life. As the themes suggested, NCDs such as diabetes generate anxiety in rural Tanzania. More community-level education is needed about diabetes to decrease fear. Indeed, participants' surprise at having diabetes indicates an overall lack of knowledge of the disease. This is not exclusive to rural Tanzania but is found worldwide (Al-Thani et al., 2018; Kayyali et al., 2019; Kumar et al., 2013).

\section{Recommendation}

This exploration of NCDs and diabetes in subSaharan Africa and rural Tanzania and the issues surrounding the use of HbA1c machines in LMICs sheds some light on the barriers that Tanzania and any partnering entity will encounter as they seek to tackle rising diabetes incidence, prevalence and diabetesrelated mortality rates. It is helpful to examine and summarize these issues in Tanzania using a traditional lens of primary, secondary and tertiary levels of prevention. At the primary prevention level, the conceptualization of terms foundational to diabetes care and NCD management are non-existent, e.g., continuity of care and chronic disease (self)-management (Ndayisaba et al., 2017). There are significant structural gaps in the health system itself, especially in rural areas and Tanzania has tremendous healthcare provider shortages (Mwangome et al., 2016). Much known about successful diabetes care in developed countries relies on baseline knowledge about diabetes, dietary changes, selfmonitoring and medication adherence. However, that model is not readily translated to the Tanzanian context, since many Tanzanians seem to have a limited understanding of diabetes and basic principles related to genetics and lifestyle modification, even if adapted to Tanzanian culture (Meiqari et al., 2019). Public health messaging about diabetes is virtually non-existent (Nnko et al., 2015).

In terms of secondary prevention, the evidence base to support diabetes screening in LMICs is not established and there are ethical questions about screening patients in order to diagnose them with a disease that cannot be cared for within such an ill-equipped system, especially 
in rural areas (Kolling et al., 2010; Atun et al., 2017). Resources needed to manage already-diagnosed diabetics (tertiary prevention) are either non-existent, unreliable, maxed out, or ineffective. There is limited knowledge about diabetes and programs are often contained in sparsely placed, underutilized diabetes initiatives within distant hospitals (Ndayisaba et al., 2017). In some settings, medical personnel, mainly clinical officers, regularly perform 45 to 50 consultations per day (Some et al., 2016). Diabetes care is not often incorporated into primary care/village clinics and providers feel inadequate to care for diabetic patients (Mwangome et al., 2016). There is a basic lack of facilities, diabetes monitoring devices, including $\mathrm{HbA1c}$ equipment and medications. Advanced diabetes care (dialysis, insulin pumps, ICU care) is often unthinkable. Diabetes complication management such as retinopathy, nephropathy or foot ulceration is largely impossible in rural sub-Saharan Africa (Price et al., 2011). For patients to seek care, diabetes care quality needs to be high and trust in the provider must exist (Nnko et al., 2015; Mwangome et al., 2016); this seems unattainable due to profound underlying structural gaps.

It is easy to get discouraged while attempting to figure out a way to bolster diabetes care efforts in rural Tanzania, once one has come to a greater understanding of the seemingly insurmountable barriers. However, there are many valid cost-effective recommendations to tackle the problem of diabetes in rural Tanzania, many of which are outlined in official NCD/diabetes care recommendations (WDF07-265, 2007; MHSW, 2008; WHO, 2017). Health officials in the Mara District and within Shirati Hospital are in agreement: Diabetes is a key area of concern. Strengthening capacity, especially at the primary level, should be a focus. This can be done by learning from the success of existing infectious disease initiative programs and through decentralization and task-shifting (shifting care to nurses and to community health workers) (Park and Pastakia, 2018). For instance, Shirati Hospital and its outreach clinics function as a district hospital and have been a sustained, successful and well-supported grass-roots partnership for almost one hundred years. A Western-style palliative care program exists as well as a successful nurse-led outreach clinic for cervical cancer, so there is precedence for nurse led programs (Shirati KMT Hospital, 2020). This indicates the possibility of adoption of (Some et al., 2016) model of incorporating diabetes care into alreadyexisting outreach clinics through staff development. Shirati is a vibrant community full of interested, engaged healthcare providers, students and patients with high care needs. The creation of nurse-led diabetes clinics as part of an American nursing school/Shirati Hospital partnership is a viable option to relieve care gaps because it relies on a strong history and leverages existing strengths: Nursing education, nurse empowerment and knowledge of chronic disease management, including diabetes. Community involvement is key to creating coordinated care, continuity and patient centeredness (Mwangome et al., 2016).

\section{Conclusion}

Although Tanzania is well-aware of the urgent need to prevent, diagnose and manage diabetes, it has created strategic plans that largely do not correspond to the reality of its profoundly under-resourced system (MHSW, 2008; WDF, 2016; WHO, 2017). Many suggestions (e.g., diabetes registry) rely on numerous health professionals and a specialized knowledge base that does not exist (e.g., diabetes educators). Many Western organizations such as disease-focused nonprofit foundations and/or universities and medical schools have a sincere desire to help but lack the ability or the time needed to invest in ongoing mentoring or resources. Tackling a double disease burden demands creativity and flexibility, as well as significant resources and readiness for global partnering (WHO, 2017).

Many successful programs in sub-Saharan Africa have been modeled after HIV care, now perceived to be a chronic disease (Atun et al., 2017; Gill et al., 2008; Nnko et al., 2015; Mwangome et al., 2017; 2016). Some et al. (2016) suggest that lessons should be learned from HIV management in mobilizing health services to manage NCDs. Nearby sub-Saharan African countries have decentralized, incorporating local, primary care-based, prevention-focused, nurse-led diabetes clinics into their health systems.

Nurse-led teams can implement global initiatives that have the ability to impact the health of individuals and communities. This is a tactic learned from HIV programs that use community health workers and nurses (Mwangome et al., 2016; Atun et al., 2017). In such clinics (Gill et al., 2008; Price et al., 2011; Meiqari et al., 2019). Basic patient education is emphasized, as is community-based prevention. Most utilized low-cost management tactics, such as foot clinics, individual education sessions, blood glucose measurement, weight, urinalysis, blood pressure and medication monitoring and follow-up. Cost-effective screening entailed identifying high-risk individuals through risk factor identification questionnaires (Peer et al., 2014). Creative solutions can include paper-based appointment systems, patient-held records, peer educators, support groups, mobile phone apps and text options (Meiqari et al., 2019). Several clinics incorporated protocols for assessment and nurse prescribing (Some et al., 2016).

With sufficient planning and preparation, $\mathrm{HbA} 1 \mathrm{c}$ monitoring can be introduced in resource-limited 
settings. Mentorship is essential (Ndayisaba et al., 2017). The Tanzania $\mathrm{HbA} 1 \mathrm{c}$ project functions as a case study through which to examine the complexities of diabetes management in a resource-constrained environment.

\section{Acknowledgement}

The authors would like to acknowledge Dr. Bwire Chirangi and the personnel at Shirati Hospital, the citizens of Shirati, Dale Ressler and DNP students Janice Gandy, Katie Skiff and Caitlin Crowder for assisting in the implementation of this project.

\section{Author's Contributions}

Andrea Fortson Knopp: Participated in all research, coordinated data analysis and contributed to writing the manuscript.

Erika Metzler Sawin: Participated in coordinated of data analysis and contributed to writing the manuscript.

\section{Ethics}

This project was undertaken with the approval of the Internal Review Board at James Madison University and Shirati Hospital with a support letter from Dr. Bwire Chirangi, Medical Director. Consent was obtained by all participants any vulnerable populations were protected.

\section{References}

Al-Thani, A. A., Farghaly, A. H., Akram, H., Khalifa, S. E., Vinodson, B., Loares, A. M., \& Abou-Samra, A. B. (2018). Public awareness and perceptions about diabetes in the State of Qatar. Cureus, 10(5).

Atun, R., Davies, J. I., Gale, E. A., Bärnighausen, T., Beran, D., Kengne, A. P., ... \& Ramaiya, K. (2017). Diabetes in sub-Saharan Africa: From clinical care to health policy. The lancet Diabetes \& endocrinology, 5(8), 622-667.

Balde, N., Camara, A., Sobngwi-Tambekou, J., Balti, E. V., Tchatchoua, A., Fezeu, L., ... \& Sobngwi, E. (2017). Improving access to HbAlc in sub-Saharan Africa (IA3) cohort: Cohort profile. The Pan African Medical Journal, 27.

Bergman, M., Buysschaert, M., Schwarz, P. E., Albright, A., Narayan, K. V., \& Yach, D. (2012). Diabetes prevention: Global health policy and perspectives from the ground. Diabetes management (London, England), 2(4), 309.

Camara, A., Baldé, N. M., Sobngwi-Tambekou, J., Kengne, A. P., Diallo, M. M., Tchatchoua, A. P., ... \& Sobngwi, E. (2015). Poor glycemic control in type 2 diabetes in the South of the Sahara: The issue of limited access to an $\mathrm{HbA1c}$ test. Diabetes research and clinical practice, 108(1), 187-192.
CDC. (2019). CDC in Tanzania. Centers for Disease Control.

https://www.cdc.gov/globalhealth/countries/tanzania /pdf/Tanzania_Factsheet-h.pdf

Drain, P. K., Hyle, E. P., Noubary, F., Freedberg, K. A., Wilson, D., Bishai, W. R., ... \& Bassett, I. V. (2014). Diagnostic point-of-care tests in resourcelimited settings. The Lancet infectious diseases, 14(3), 239-249.

Gill, G. V., Price, C., Shandu, D., Dedicoat, M., \& Wilkinson, D. (2008). An effective system of nurse-led diabetes care in rural Africa. Diabetic Medicine, 25(5), 606-611.

Kayyali, R., Slater, N., Sahi, A., Mepani, D., Lalji, K., \& Abdallah, A. (2019). Type 2 Diabetes: how informed are the general public? A cross-sectional study investigating disease awareness and barriers to communicating knowledge in high-risk populations in London. BMC public health, 19(1), 138. doi:10.1186/s12889-019-6460-7

Kolling, M., Winkley, K., \& von Deden, M. (2010). "For someone who's rich, it's not a problem". Insights from Tanzania on diabetes health-seeking and medical pluralism among Dar es Salaam's urban poor. Globalization and Health, 6(1), 8 .

Kumar, A., Goel, M. K., Jain, R. B., Khanna, P., \& Chaudhary, V. (2013). India towards diabetes control: Key issues. The Australasian medical journal, 6(10),524-531.

DOI: 10.4066/AMJ.2013.1791

Meiqari, L., Al-Oudat, T., Essink, D., Scheele, F., \& Wright, P. (2019). How have researchers defined and used the concept of 'continuity of care'for chronic conditions in the context of resourceconstrained settings? A scoping review of existing literature and a proposed conceptual framework. Health research policy and systems, 17(1), 27.

Mwangome, M. N., Geubbels, E., Klatser, P., \& Dieleman, M. (2016). "I don't have options but to persevere." Experiences and practices of care for HIV and diabetes in rural Tanzania: A qualitative study of patients and family caregivers. International journal for equity in health, 15(1), 1-13.

Mwangome, M., Geubbels, E., Klatser, P., \& Dieleman, M. (2017). Perceptions on diabetes care provision among health providers in rural Tanzania: A qualitative study. Health policy and planning, 32(3), 418-429.

Ndayisaba, A., Harerimana, E., Borg, R., Miller, A. C., Kirk, C. M., Hann, K., ... \& Mutumbira, C. (2017). A clinical mentorship and quality improvement program to support health center nurses manage type 2 diabetes in rural Rwanda. Journal of diabetes research, 2017. 
Nnko, S., Bukenya, D., Kavishe, B. B., Biraro, S., Peck, R., Kapiga, S., ... \& Seeley, J. (2015). Chronic diseases in North-West Tanzania and Southern Uganda. Public perceptions of terminologies, aetiologies, symptoms and preferred management. PloS one, 10(11), e0142194.

Park, P. H., \& Pastakia, S. D. (2018). Access to Hemoglobin A1c in rural Africa: A difficult reality with severe consequences. Journal of diabetes research, 2018.

Peer, N., Kengne, A. P., Motala, A. A., \& Mbanya, J. C. (2014). Diabetes in the Africa Region: An update. Diabetes research and clinical practice, 103(2), 197-205.

Peprah, S., Dhudha, H., Ally, H., Masalu, N., Kawira, E., Chao, C. N., ... \& Otim, I. (2019). A population-based study of the prevalence and risk factors of low-grade Plasmodium falciparum malaria infection in children aged $0-15$ years old in northern Tanzania. Tropical Medicine \& International Health, 24(5), 571-585.

Price, C., Shandu, D., Dedicoat, M., Wilkinson, D., \& Gill, G. V. (2011). Long-term glycaemic outcome of structured nurse-led diabetes care in rural Africa. QJM: An International Journal of Medicine, 104(7), 571-574.

Shirati KMT Hospital. (2020). About Shirati. Shitati KMT http://shiratihospital.org/index.php/en/

Some, D., Edwards, J. K., Reid, T., Van den Bergh, R., Kosgei, R. J., Wilkinson, E., ... \& Kibachio, J. (2016). Task shifting the management of noncommunicable diseases to nurses in Kibera, Kenya: Does it work?. PLoS One, 11(1), e0145634.

Stanifer, J. W., Cleland, C. R., Makuka, G. J., Egger, J. R., Maro, V., Maro, H., ... \& Philippin, H. (2016). Prevalence, risk factors and complications of diabetes in the Kilimanjaro region: A population-based study from Tanzania. PloS one, 11(10), e0164428.
MHSW. (2008). National noncommunicable disease strategy. The United Republic of Tanzania Ministry of Health and Social Welfare https://www.iccpportal.org/system/files/plans/Tanzania_National\%20 \%20NCD\%20strategy_2008-1.pdf

Wetzel, T.L. (2011). Western and traditional medicine use practices in Shirati, Tanzania https://pdfs.semanticscholar.org/f454/5d6f6216f03d b594bbd3e5854b2b0fb9bde3.pdf

WDF. (2016). Strategic and action plan for the prevention and control of non- communicable diseases in Tanzania 2016-2020. https:/www.worlddiabetesfoundation.org/sites/defa ult/files/NCD\%20Stategic\%20Plan\%202016\%20$\% 202020 . p d f$

WDF07-265. (2007) Diabetes awareness, care and referral in Lake Zone - a comprehensive model of care WDF07-265; Strengthening the capacity for diabetes care in the Mara, Kagera and Shinyanga regions in Northern Tanzania. https://www.worlddiabetesfoundation.org/projects/ta nzania-wdf07-265.

WHO. (2012). STEPwise approach to chronic disease risk factor surveillance, Tanzania. World Health Organization.

https://www.who.int/ncds/surveillance/steps/UR_Ta nzania_2012_STEPS_Report.pdf

WHO. (2016). Diabetes country profiles: Tanzania. World Health Organization. Accessed 16 June 2019, https: www.who.int/diabetes/countryprofiles.

WHO. (2017). Primary health care systems (PRIMASYS): Comprehensive case study from United Republic of Tanzania. Geneva: World Health Organization. https://www.who.int/alliancehpsr/projects/AHPSR-PRIMASYS-Tanzaniacomprehensive.pdf?ua $=1$ 